\title{
Melanin Is Not Required for Turgor Generation but Enhances Cell-Wall Rigidity in Appressoria of the Corn Pathogen Colletotrichum graminicola
}

\author{
Nancy Ludwig, ${ }^{1}$ Marco Löhrer, ${ }^{2}$ Marcus Hempel, ${ }^{3}$ Sebastian Mathea, ${ }^{4}$ Ivo Schliebner, ${ }^{2}$ \\ Matthias Menzel, ${ }^{5}$ Andreas Kiesow, ${ }^{5}$ Ulrich Schaffrath, ${ }^{2}$ Holger B. Deising, ${ }^{1,3}$ and Ralf Horbach ${ }^{3}$ \\ ${ }^{1}$ Institute for Agricultural and Nutritional Sciences, Martin-Luther-University Halle-Wittenberg, Betty-Heimann-Str. 3 , \\ D-06120 Halle (Saale), Germany; ${ }^{2}$ Department of Plant Physiology (Biology III), RWTH Aachen University, D-52056 \\ Aachen, Germany; ${ }^{3}$ Interdisciplinary Center for Crop Plant Research, Martin-Luther-University Halle-Wittenberg, Betty- \\ Heimann-Str. 3, D-06120 Halle (Saale), Germany; ${ }^{4}$ Max Planck Research Unit for Enzymology of Protein Folding, \\ Weinbergweg 22, D-06120 Halle (Saale), Germany; ${ }^{5}$ Fraunhofer Institute for Mechanics of Materials, Walter-Hülse-Str. 1, \\ D-06120 Halle (Saale), Germany
}

Submitted 12 September 2013. Accepted 11 November 2013.

The ascomycete and causative agent of maize anthracnose and stem rot, Colletotrichum graminicola, differentiates melanized infection cells called appressoria that are indispensable for breaching the plant cell wall. High concentrations of osmolytes accumulate within the appressorium, and the internal turgor pressure of up to 5.4 MPa provides sufficient force to penetrate the leaf epidermis directly. In order to assess the function of melanin in $C$. graminicola appressoria, we identified and characterized the polyketide synthase 1 (CgPKS1) gene which displayed high similarity to fungal polyketide synthases (PKS) involved in synthesis of 1,3,6,8-tetrahydronaphthalene, the first intermediate in melanin biosynthesis. Cgpks 1 albino mutants created by targeted gene disruption were unable to penetrate intact leaves and ruptured frequently but, surprisingly, were able to penetrate ultrathin polytetrafluoroethylene membranes mimicking the plant surface. Nonmelanized Cgpks1 appressoria were sensitive to externally applied cell-wall-degrading enzymes whereas melanized appressoria were not affected. Expression studies using a truncated $C g P K S 1$ fused to green fluorescent protein revealed fluorescence in immature appressoria and in setae, which is in agreement with transcript data obtained by RNA-Seq and quantitative polymerase chain reaction. Unexpectedly, surface scans of mutant and wild-type appressoria revealed considerable differences in cell-wall morphology. Melanization of appressoria is indispensable for successful infection of intact leaves. However, cell collapse experiments and analysis of the appressorial osmolyte content by Mach-Zehnder interferometry convincingly showed that melanin is not required for solute accumulation and turgor generation, thus questioning the role of melanin as a barrier for osmolytes in appressoria of $C$. graminicola.

The nucleotide sequence data reported has been deposited in the GenBank database (accession number FJ194435).

Corresponding author: R. Horbach; E-mail: ralf.horbach@landw.uni-halle.de

* The $\boldsymbol{e}$-Xtra logo stands for "electronic extra" and indicates that nine supplementary figures are published online.

(C) 2014 The American Phytopathological Society
Melanins are chemically diverse, dark pigments composed of polymerized phenolic or indolic compounds. They are produced by a broad variety of organisms, including mammals, insects, and microbes. Among the multiple proposed functions, protection against radiation, heavy metals, xenobiotics, phagocytosis, desiccation, osmolytes, hydrolytic enzymes, oxidatives, heat, and cold are regarded as the most prevalent (Henson et al. 1999; Liu and Nizet 2009; Nosanchuk and Casadevall 2006).

Among several different types of melanin, the most important types identified in fungi are dihydroxynaphthalene (DHN) melanin and L-3,4-dihydroxyphenylalanine (DOPA) melanin. The first traceable product of the DHN pathway is 1,3,6,8-tetrahydroxynaphthalene (THN), which originates from five acetate units. THN is subsequently converted to DHN via the intermediates scytalone, 1,3,8-trihydroxynaphthalene, and vermelone. Finally, DHN monomers polymerize in the apoplast by a process that may involve free radicals (Bell and Wheeler 1986; Butler and Day 1998).

Tyrosine-derived L-DOPA is the characteristic precursor in the second fungal melanin synthesis pathway. The monomeric product 5,6-indolequinone is synthesized from L-DOPA via the intermediates dopaquinone, leucodopachrome, dopachrome, and dihydroxyindole (Tian et al. 2003; Zhong et al. 2008).

Although fungal melanins have been subject to research for decades, relatively little is known about the polymeric structure of the melanin meshwork after incorporation into fungal cell walls. Only recently, employment of solid-phase nuclear magnetic resonance delivered first insights into this process. Using ${ }^{13} \mathrm{C}$-enriched precursors of melanin synthesis in Cryptococcus neoformans, Zhong and associates (2008) found components derived from cell-wall polysaccharides covalently linked to DOPA melanin, suggesting strong ties between melanin and cell-wall components, with implications for mechanical properties and enzymatic accessibility of melanized fungal cell walls.

Fungal melanins do not only serve as protectants against environmental stresses but also determine virulence in some pathogenic fungi. The mechanism by which melanin contributes to virulence has been most extensively studied in plant pathogens and fungi affecting human health (Henson et al. 1999; Idnurm and Howlett 2001; Liu et al. 2008; Nosanchuk and Casadevall 2006; Revankar and Sutton 2010; Youngchim et al. 2011).

Vol. 27, No. 4, 2014 / 315 
In the human pathogens Aspergillus fumigatus and C. neoformans, melanin was shown to confer protection against recognition by the innate immune system (Chai et al. 2010; Thywißen et al. 2011; Volling et al. 2011) and increased resistance to phagocytic killing (Mednick et al. 2005; Wang et al. 1995).

Experimental data provide evidence that melanin acts as a determinant of virulence or pathogenicity in specialized infection structures of plant-pathogenic fungi. Appressoria of the apple scab fungus Venturia inaequalis are distinguished by a melanized appressorial ring structure (MARS) precisely located around the penetration pore. The application of melanin biosynthesis inhibitors prevents the formation of MARS and leads to a significantly decreased penetration rate (Steiner and Oerke 2007). Because extracted melanin of $V$. inaequalis was shown to be associated with secreted hydrolases that are slowly released, appressorial melanin may serve to concentrate cell-wall-degrading enzymes (CWDE) at the penetration site (Hignett et al. 1978). Interestingly, a study by Gachoma and associates (2010) describes the formation of corresponding ring structures in appressoria of Diplocarpon rosae, a pathogen causing black spot disease of rose leaves.

Melanin biosynthesis is also required for full virulence of Gaeumannomyces graminis, an economically important root pathogen of wheat. Infected cereal roots are surrounded by dark infection cushions that are composed of clustered hyphopodia, which share a similar morphology with appressoria but originate from vegetative hyphae rather than from germ tubes (Freeman and Ward 2004). A G. graminis albino mutant was shown to be nonpathogenic, and the DHN melanin synthesis inhibitors tricyclazole and PP389 reduced the virulence of the wild type (WT) (Kelly et al. 1997). Money and associates (1998) demonstrated that hyphopodia of the WT strain generate higher pressure than nonmelanized hyphopodia, and melanization of the hyphopodial cell wall was associated with an increase in its rigidity.

Fungal plant pathogens such as Magnaporthe oryzae and Colletotrichum spp. penetrate plant barriers by generating an exceptionally high turgor pressure in melanized appressoria that drives the penetration peg through leaf cuticles and cell walls. Melanin-deficient mutants of these species are nonpathogenic due to impaired penetration competence.

According to the current hypothesis, the melanin layer forms a narrow meshwork adjacent to the cell membrane in appressoria of these fungi, thereby limiting cell-wall permeability to retain low molecular weight osmolytes. As a consequence, high concentrations of osmolytes accumulate in the appressorium, which leads to a hydrostatic turgor pressure of up to $8 \mathrm{MPa}$, thereby generating sufficient force to penetrate the plant cuticle or artificial membranes (Freeman and Ward 2004; Henson et al. 1999; Howard et al. 1991; Loehrer et al. in press; Money 1995).

The current concept of the function of melanized appressoria of $M$. oryzae assumes that pore sizes below the effective diameter of glycerol are formed by the incorporation of melanin, which was shown to be the major osmolyte in this fungus (DeJong et al. 1997). However, consistent cross-linking that makes cell walls effective barriers for small molecule osmolytes is still discussed because radical polymerization appears to be a random process that includes cell-wall components and melanin precursors (Zhong et al. 2008). Based on the solute exclusion technique, pore diameters in cell walls of melanized appressoria of $M$. oryzae have been estimated to be less than $1 \mathrm{~nm}$ (Howard et al. 1991). The effective hydrodynamic radius of glycerol, however, is $0.31 \mathrm{~nm}$ (Miner and Dalton 1953). Consistent cross-linking based on radical polymerization of DHN monomers and cell-wall sugar alcohols, which leads to pore diameters with an exclusion threshold between water molecules and glycerol, appears to be challenging. Importantly, a recent study by Loehrer and associates (in press) identified C6-sugar alcohols as major osmolytes in addition to glycerol in appressoria of M. oryzae.

In order to understand the role of melanin in appressoria of the maize anthracnose fungus Colletotrichum graminicola, we functionally characterized the polyketide synthase 1 ( $C g P K S 1)$ gene encoding an iterative type I polyketide synthase (PKS) that produces the melanin precursor THN. When inoculated onto maize leaves, conidia of this fungus germinate and differentiate dome-shaped melanized infection cells called appressoria (Bergstrom and Nicholson 1999; Deising et al. 2000). Synthesis and accumulation of high concentrations of solutes within these specialized infection cells generates an enormous turgor pressure corresponding to approximately 5.5 $\mathrm{MPa}$, which is translated into a force of approximately $17 \mu \mathrm{N}$ (Bastmeyer et al. 2002; Bechinger et al. 1999). This force drives the penetration peg through the host cuticle and epidermal cell wall. Upon successful invasion of the epidermal cell, biotrophic infection vesicles and primary hyphae are formed. Following the initial biotrophic period, perception of as-yet-unknown signals leads to a switch in lifestyle and formation of fastgrowing necrotrophic secondary hyphae, which rapidly colonize and kill the host tissue (Bergstrom and Nicholson 1999; Horbach et al. 2011).

In this study, we reexamined the proposed function of melanin in appressoria of $C$. graminicola. Based on the data presented here, we show that the type I PKS CgPKS1 of the maize pathogen $C$. graminicola is required for melanin synthesis and penetration of the host epidermis but not for appressorial turgor generation and penetration of artificial membranes.

\section{RESULTS}

Isolation of $C g P K S 1$, in vitro phosphopantetheinylation, and expression studies.

A small fragment of the $C g P K S 1$ open reading frame (ORF) was isolated previously by subtractive hybridization employed to identify genes of $C$. graminicola that are upregulated in the early stage of maize infection (Sugui and Deising 2002). The complete DNA sequence of $C g P K S 1$, including an approximately 6-kbp flanking region upstream and downstream of the ORF of $C g P K S 1$, was obtained by genome walking, according to Liu and Baird (2001). Comparative analysis of the genomic and cDNA sequences revealed a 6,706-bp ORF, interrupted by three introns of 65,50 , and $54 \mathrm{bp}$, respectively (Supplementary Fig. S1A). The deduced protein consists of 2,178 amino acids (aa), corresponding to a molecular mass of $237 \mathrm{kDa}$. The protein shares significant identity with other type I PKS referred to as THN or melanin synthases (e.g., 90, 47, and 71\% identity with the PKS1 proteins of $C$. lagenarium, Bipolaris oryzae, and the M. oryzae ALB1 protein, respectively).

Type I PKS require post-translational modification by sfptype $4^{\prime}$-phosphopantetheinyl transferases (Walsh et al. 1997). In vitro $4^{\prime}$-phosphopantetheinylation of a 100-aa fragment of CgPKS1 (CgPKS100) containing the ACP domain with a conserved serine that serves as phosphopantetheinyl (PP)acceptor demonstrated substrate function of CgPKS1 in the phosphopantetheinyl transferase (CgPPT1; GenBank accession number DQ028305) catalyzed reaction, resulting in activation of PKS1 (Elovson and Vagelos 1968; Walsh et al. 1997).

Expression of a truncated CgPKS1 fused in frame to green fluorescent protein (GFP) (CgPKS:GFP) under control of the $C g P K S 1$ promoter clearly showed that the appearance of GFP fluorescence coincides with the onset of melanization in both 
appressoria and setae. Because fluorescence appears to be evenly distributed across appressoria and setae, the fusion construct is most likely directed to the cytoplasm (Fig. 1A).

Quantitative reverse-transcriptase polymerase chain reaction (qRT-PCR) using specific primers for the amplification of a 152-bp CgPKS1 fragment revealed a maximum of transcript abundance at 12 to $24 \mathrm{~h}$ postinoculation (hpi), which correlates with the initiation of melanization in appressoria (Fig. 1B). However, significant amounts of CgPKS1 transcripts could be detected at later stages of maize infection, which may be explained by the poorly synchronized differentiation of melanized appressoria. Lower transcript levels at late stages of the infection process reflect CgPKS1 activity that leads to melanization of setae. Because setae are rare acicular structures confined to acervuli, the abundance of $C g P K S 1$ transcripts is extremely low compared with the whole RNA population. Thus, after normalization, qRT-PCR data obtained from total RNA from infected leaves indicated relatively low CgPKS1 transcript abundance during the late infection stage, although fluorescing setae convincingly showed CgPKS1 expression within these specialized structures (Fig. 1).

In addition to qPCR experiments, we performed wholegenome transcript analysis (RNA-seq). The transcript profiles of genes involved in biosynthesis and regulation of melanin precursors (i.e., putative hydroxynaphthalene reductases, scytalone dehydratase, and transcription factors) displayed highest transcript abundances at the time of appressorium maturation at 12 hpi followed by a steep decline (Supplementary Fig. S3).
Targeted disruption of $C g P K S 1$ and genetic complementation of $C g p k s 1$ strains.

To functionally characterize the $C g P K S 1$ gene, targeted mutagenesis was performed. In the disruption vector, the 3.7kb Bsp119I fragment carrying the Escherichia coli hygromycin $(h p h)$ phosphotransferase cassette was integrated into the CgPKS1 ORF at nucleotide 4,415 (Supplementary Fig. S4A). As indicated by Southern blot analysis performed with XhoIdigested genomic DNA, the 6,170-bp WT band had been replaced by an 8,780-bp fragment in all independent disruption (Cgpks l) (knock-out [KO]) strains. Transformants with an ectopically integrated $\mathrm{KO}$ vector showed a band above $10 \mathrm{kbp}$, in addition to the 6,170-bp WT band. To confirm gene inactivation at the transcript level, RT-PCR experiments were performed with primers specific for $C g P K S 1$ transcripts using total RNA from WT and three independent KO strains. Primers corresponding to transcripts of the constitutively expressed chitin synthase gene $C g C H S I I$ (Werner et al. 2007) were used in a control reaction. As expected, the 380-bp $\mathrm{Cg} C H S I I$ fragment was amplified from RNA from WT and KO strains but the 224-bp CgPKS1 fragment only occurred when RNA from the WT strain was used as a template. These data confirm successful targeted inactivation of $C g P K S 1$.

All Cgpks 1 mutants generated lack melanization and appear yellow to light orange on solid medium whereas the WT and a Cgpks1 strain complemented with the full-length $C g P K S 1$ gene exhibit melanized sclerotial tissue and appressoria (Fig. $2 \mathrm{~A}$ and B). Complementation of Cgpks1 with $C g P K S 1$ under
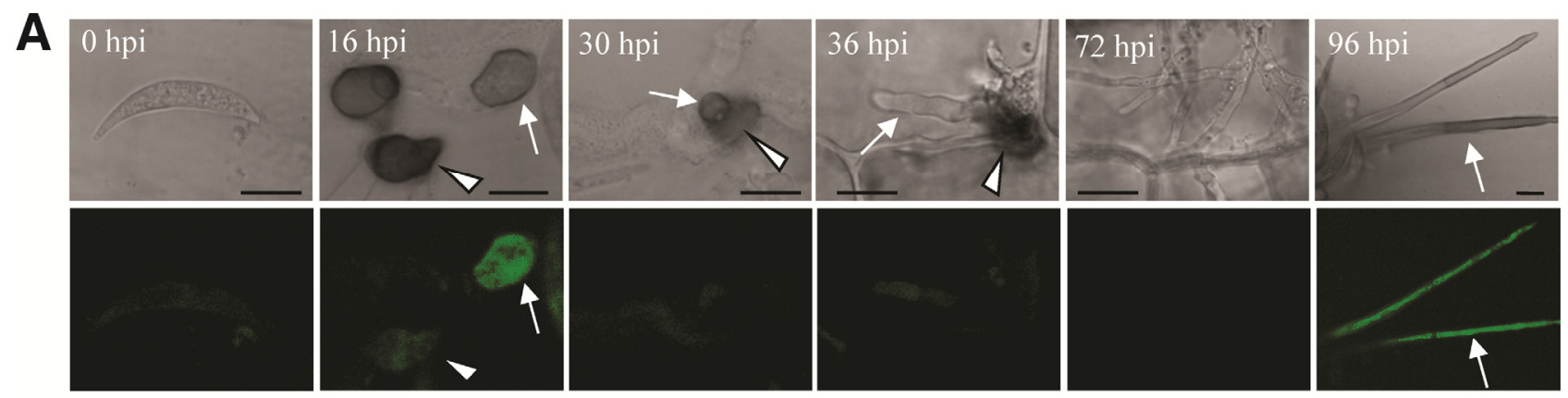

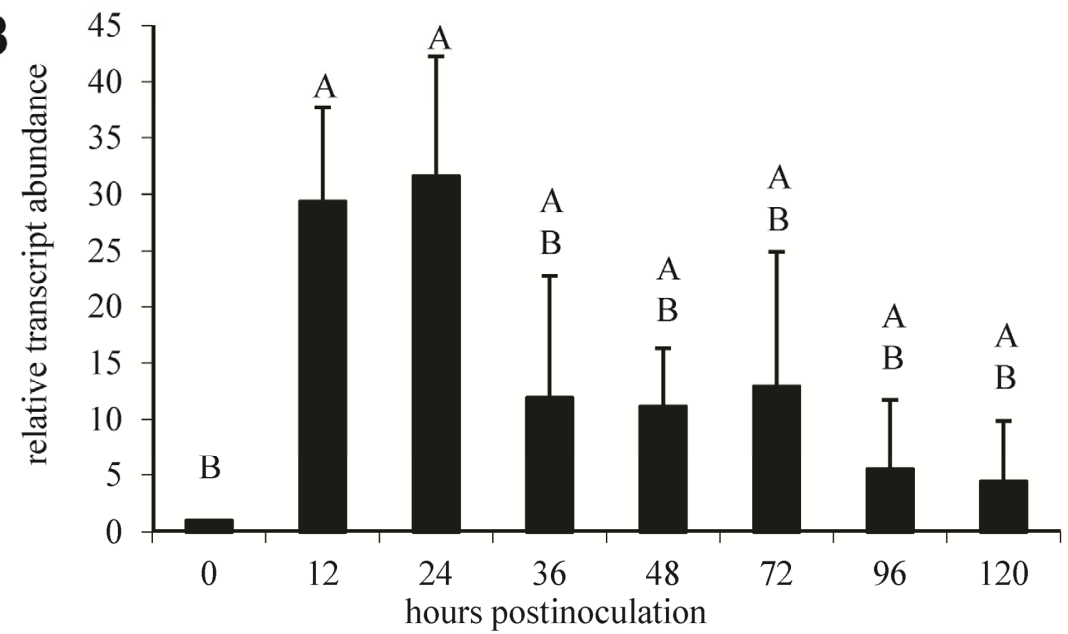

Fig. 1. Expression of CgPKS1 during pathogenic development of Colletotrichum graminicola. A, Expression of a PKS1:GFP fusion construct in infected maize leaves. By $16 \mathrm{~h}$ postinoculation (hpi), appressoria had formed on the plant surface (16 hpi, arrow). At 30, 36, 72 , and 96 hpi, infection vesicles, primary hyphae, secondary hyphae, and acervuli with setae had differentiated as indicated by arrows. GFP fluorescence is visible in immature appressoria (16 hpi, arrows) but not in mature appressoria with full melanization (16 hpi, arrowheads). GFP fluorescence in setae became visible with the onset of melanization (96 hpi, arrows). Arrowheads at 30 and 36 hpi indicate the position of the appressorium. Bright-field microscopy images are shown in the upper row and the respective confocal fluorescence images are shown in the lower row. Size bars $=10 \mu \mathrm{m}$. B, Quantitative reverse-transcriptase polymerase chain reaction (qRT-PCR) analysis of the $C g P K S 1$ transcript level. Relative amounts of the $C g P K S 1$ transcripts determined by qRT-PCR at eight time points of the infection course of maize leaves. Bars indicate standard deviations of three biological repeats. 
control of the constitutive oliC promoter resulted in heavily melanized sclerotial tissue (Fig. 2A).

Because a functional $C g P K S 1$ is essential for melanization of appressoria and pathogenicity in some phytopathogenic fungi, we analyzed the role of this gene in maize infection assays. When inoculated onto nonwounded leaves, the WT but not the Cgpks1 mutant was able to cause anthracnose symptoms (Fig. 2C). Although appressorial cell walls of both complementation strains displayed somewhat reduced melanization as compared with the WT, they regained full virulence on intact maize leaves (Fig. 2C). In contrast, addition of scytalone, a metabolite of the melanin biosynthesis pathway downstream of THN, resulted in melanization of appressoria but distribution of melanin within the appressorial cell wall appeared irregular. The cell-wall rigidity of these appressoria was considerably reduced, as indicated by deformed or ruptured appressoria (Supplementary Fig. S5).

Melanin-deficient mutants of $C$. graminicola obtained after UV irradiation have been shown to be impaired in host cell penetration (Rasmussen and Hanau 1989). In order to confirm this observation in our experimental system we, compared the penetration competence of WT and Cgpksl appressoria on maize leaves. Conidia of two individual Cgpksl strains sprayed onto intact leaves germinated and differentiated appressoria within $24 \mathrm{hpi}$ at rates comparable with those of the WT. Germination rates of WT and Cgpksl conidia were $72 \pm 5.3$ and $63.3 \pm 6.8 \%$, respectively, and $52.0 \pm 8.5 \%$ of the WT and $47.0 \pm 6.6 \%$ of the Cgpks 1 germ tubes differentiated appressoria. Although $86.3 \pm 8.0 \%$ of the WT appressoria successfully penetrated the maize epidermis and subsequently formed infection vesicles and primary hyphae in planta, none of the nonpigmented appressoria differentiated by the Cgpks strains were able to penetrate the leaf epidermis (Fig. 3A). Moreover, whereas Cgpks1 appressoria frequently ruptured on the leaf surface, WT appressoria remained structurally intact, and their cytoplasmic content had been transferred into the in planta differentiated infection structures (Fig. 3; Supplementary Fig. S8). On wounded leaves, however, both the WT and the Cgpks 1 strains efficiently penetrated anticline plant cell walls, colonized the leaf tissue, and caused anthracnose symptoms. These results clearly show divergent penetration mechanisms of external and anticlinal host cell walls.

The term lateral germination describes the atypical formation of germ tubes from appressoria which may occur in consequence of a failed penetration attempt. On maize leaf segments, $90.3 \pm 3.1 \%$ of the WT conidia differentiated a single appressorium. Formation of a second appressorium from the same conidium due to lateral germination was observed at rates of $9.7 \pm 3.1 \%$. Three or more appressoria originating from one conidium could not be found for the WT strain. In contrast, multiple lateral germination events of Cgpks1 appressoria appeared frequently. Only $16.5 \pm 3.3 \%$ of the germinated Cgpkslconidia produced a single appressorium whereas the majority of $47.5 \pm 9.3 \%$ displayed a single lateral germination event leading to the formation of a second appressorium. Remarkably, three or even four appressoria differentiated by conidia of the Cgpks1 strain were found at rates of $35.3 \pm 6.7$ and $0.8 \pm 1.0 \%$, respectively (Fig. 3B).

Taken together, primary infection of intact plant surfaces depends on melanized appressoria whereas the penetration of anticline cell walls, a prerequisite for the invasive growth, is independent of melanized fungal infection structures. Complementation with scytalone rendered partially melanized appressoria that ruptured frequently on plastic slides, indicating a structural function of melanin in appressorial cell walls of C. graminicola.

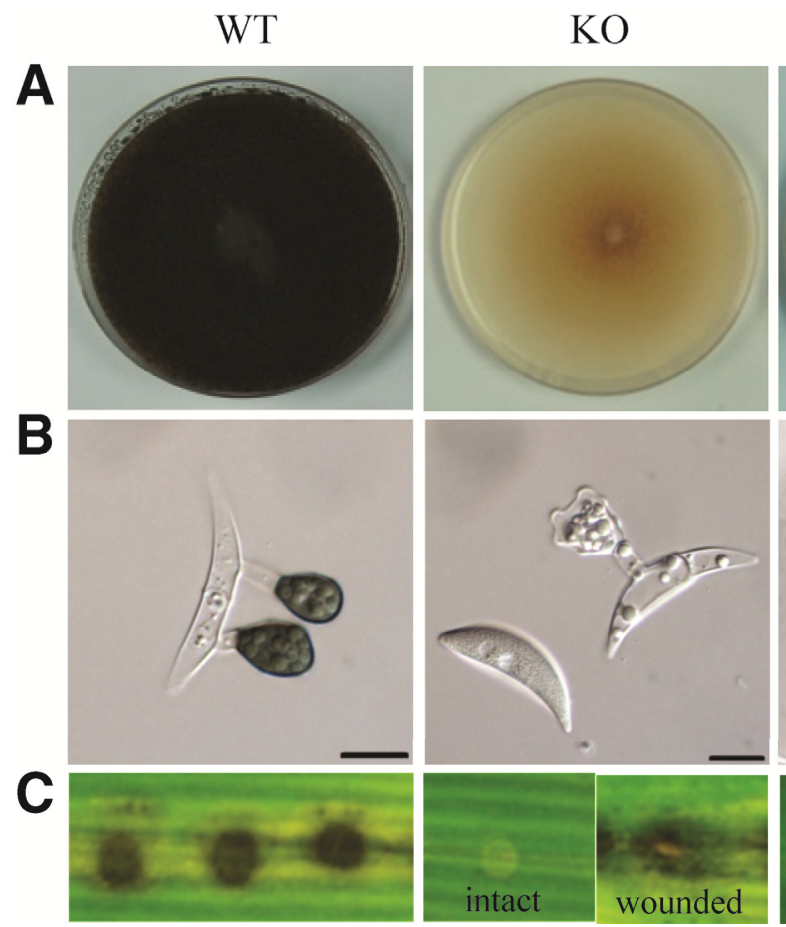

COM1 (nat)
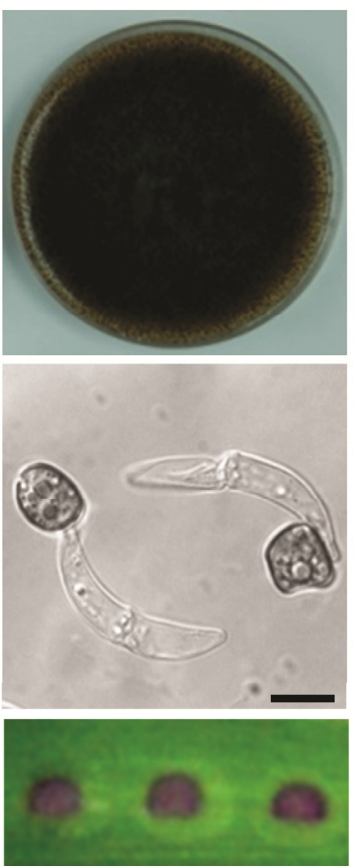

\section{COM2 (olic)}

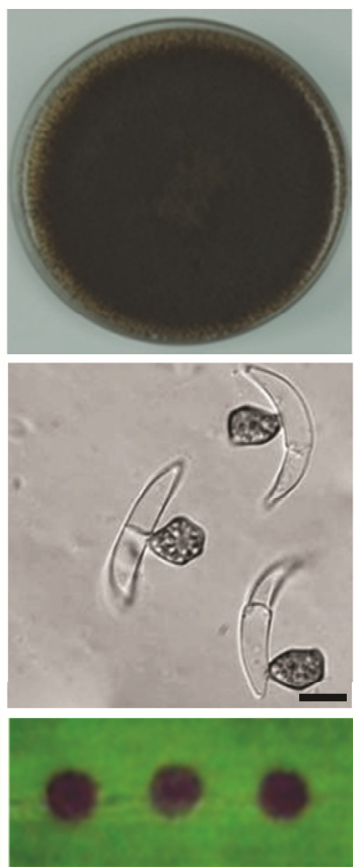

Fig. 2. Phenotype of Colletotrichum graminicola wild-type (WT), Cgpks1, and complementation (COM) strains. A, Vegetative growth of WT, CgPKS1 (knock-out [KO]), and CgPKS1 COM strains (COM1 and COM2) on oat meal agar. The absence of melanin in vegetative hyphae of the Cgpks1 strain leads to yellow to orange mycelia. Reintegration of $C g P K S 1$ under control of either the native (COM1) or the constitutive oliC promoter (COM2) into Cgpks1 restores melanization of vegetative hyphae. B, Appressoria differentiation and morphology. Appressoria produced by the C. graminicola WT are heavily melanized spherical structures. In contrast, appressoria of the Cgpks1 strain (KO) are distinguished by the complete loss of visible melanin. Cell walls of appressoria differentiated by the COM strains (COM1 and COM2) display slightly reduced melanization compared with the WT. Size bars $=10 \mu \mathrm{m}$. C, Plant infection and macroscopic evaluation of anthracnose symptom development at 6 days postinoculation. The WT but not the Cgpks1 (KO) strain cause anthracnose disease symptoms in maize leaves. Virulence was restored in the Cgpks1 strain complemented with the CgPKS1 gene (COM1 and COM2). 
In order to examine the contribution of melanin to the resistance of appressorial cell walls toward enzymatic degradation, CWDE from Trichoderma harzianum have been used to lyse structural cell-wall polymers such as 1,3-glucans and chitin. A conidium that ruptured following the treatment with lysing en- zymes of T. harzianum is depicted in Supplementary Fig. S9. Cytoplasmic lipid bodies stained with Nile Red were released and the entire cell collapsed, due to the loss of turgor. Calcofluor staining in combination with confocal laser-scanning microscopy allowed discrimination between turgescent cells, the
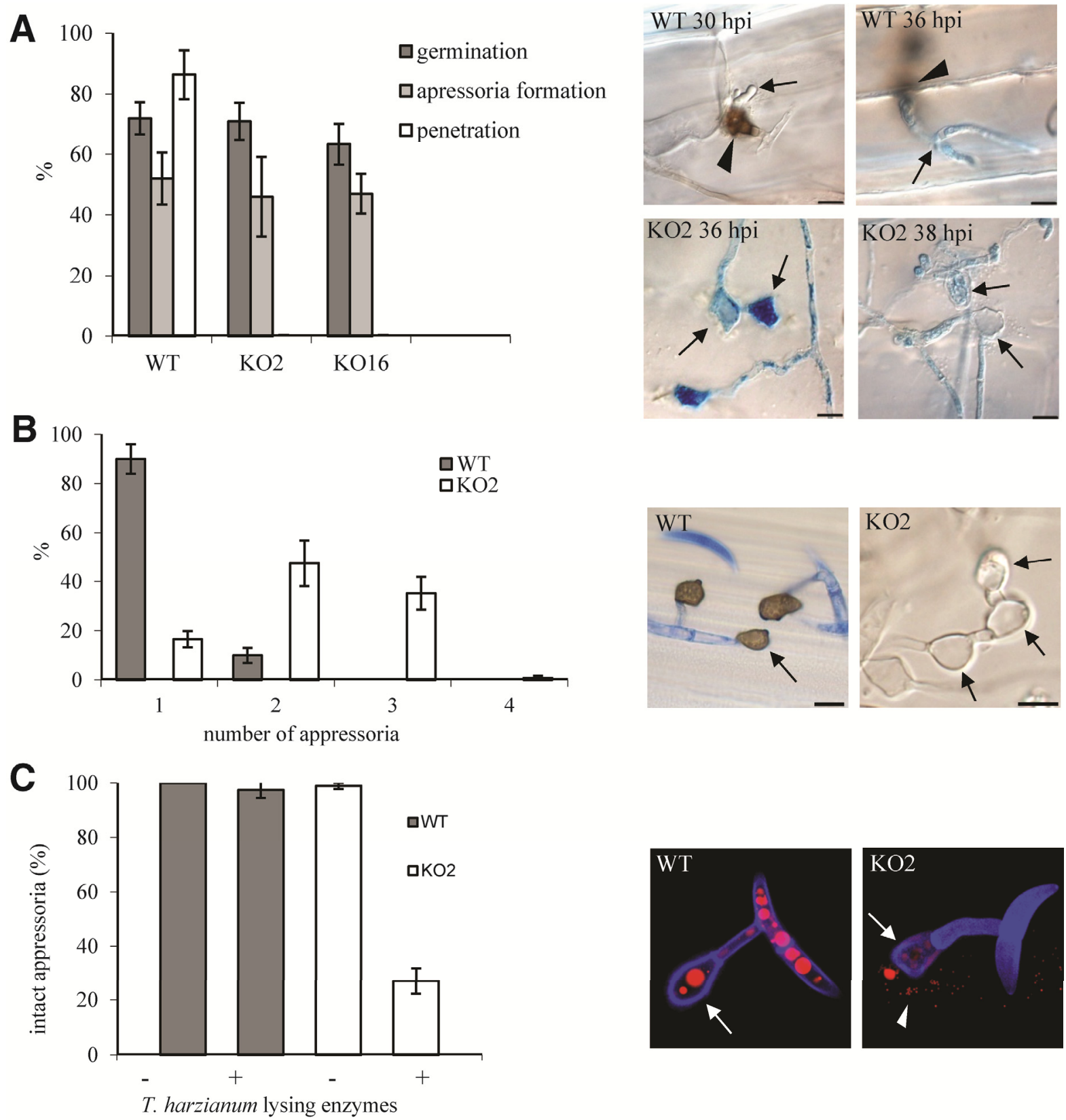

Fig. 3. Quantification of parameters affecting virulence and viability of the wild-type (WT) and melanin-deficient Cgpks1 strains. A, Leaf infection assay. Conidial germination, appressoria formation, and maize penetration rates were determined by microscopic analysis of the infection process using the WT and two individual Cgpks1 strains (KO2 and KO16). No significant differences in conidial germination and appressoria formation rates could be observed. Approximately $90 \%$ of the appressoria differentiated by the WT penetrated the maize epidermis, as indicated by the formation of apoplastic infection vesicles and biotrophic primary hyphae (WT $30 \mathrm{~h}$ postinoculation [hpi] arrow indicates an emerging biotrophic hypha, arrowhead points at the appressorium; WT 36 hpi arrow points at a branching biotrophic hypha). No successful penetration events could be observed for nonpigmented appressoria of the $C g p k s 1$ strain (KO2 36 hpi, arrows). Cgpks1 strains ruptured frequently on maize leaves, as indicated by the extrusion of cytoplasm (KO2 38 hpi). Appressoria formation rates refer to the number of germinated conidia and successful penetration events to the number of differentiated appressoria, respectively. Infected leaves were stained with aniline blue, except KO2 $38 \mathrm{hpi}(n=300$, size bars $=10 \mu \mathrm{m})$. B, Quantification of lateral germination events of appressoria differentiated by the WT and a Cgpks 1 (KO2) strain on maize leaves. Less than $10 \%$ of the WT appressoria displayed lateral germination and differentiated a second appressorium whereas the majority of the Cgpks 1 appressoria germinated and produced a second or third appressorium (arrows). Up to four appressoria differentiated by a single conidium of the Colletotrichum graminicola strain could be observed in rare cases. The WT infection sample was stained with aniline blue $(n=300$, size bars $=10 \mu \mathrm{m})$. C, Effect of cell-wall-degrading enzymes on the integrity of appressorial cell walls. Mature appressoria of the WT and a Cgpks1 (KO2) strain were incubated in a solution containing lysing enzymes of Trichoderma harzianum. Approximately $70 \%$ of the appressoria (KO2, arrow) differentiated by the KO2 strain ruptured after an incubation of $24 \mathrm{~h}$, as indicated by the extrusion of the cytoplasmic content (KO2, arrowhead). whereas the vast majority of melanized appressoria of the WT (arrow) remained intact. Samples were double-stained with Calcofluor and Nile Red $(n=150$, size bars $=10 \mu \mathrm{m})$. 
cell walls of which appeared as thin blue lines, and collapsed cells, where the entire envelope became visible. The vast majority of nonmelanized Cgpks 1 appressoria disintegrated following the treatment with lysing enzymes whereas melanized appressoria of the WT strain remained intact (Fig. 3C). Collectively, these data show that failure of penetration of Cgpks 1 appressoria led to multiple lateral germination events on the surface of maize leaves. Nonmelanized appressoria disintegrated after treatment with lysing enzymes, indicating a protective role of melanin in appressoria of $C$. graminicola.

\section{Penetration of polytetrafluoroethylene membranes,} cytorrhysis assay, and Mach-Zehnder interferometry.

Polytetrafluoroethylene (PTFE) membranes of highly defined thickness of 70 to $130 \mathrm{~nm}$ were employed to investigate the penetration competence of WT and Cgpks1 appressoria. PTFE membranes of $70 \mathrm{~nm}$ in thickness were penetrated by $19.7 \pm$ $5.8 \%$ of the WT appressoria and $30.3 \pm 17.8 \%$ of the appressoria differentiated by the Cgpks1 strain. On 100-nm-thick PTFE layers, a significantly reduced penetration rate was observed in both the WT $(0.3 \pm 0.5 \%)$ and the Cgpksl strain $(0.5 \pm 0.9 \%)$, and neither WT nor Cgpks1 appressoria were able to penetrate PTFE layers of $130 \mathrm{~nm}$ (Fig. 4A). Successful penetration of PTFE membranes by the melanin-deficient Cgpks 1 strain was visualized by electron microscopy (Fig. 4A, pictures $\mathrm{L}$ and $\mathrm{R}$ ).

Determination of incipient cytorrhysis is a valuable tool for estimation of the osmotic potential of appressoria (Howard et al. 1991). Increasing concentrations of high molecular weight osmolytes such as polyethylene glycol (PEG)-6000, which cannot permeate through the appressorial cell wall, cause visible cell collapse as soon as the external osmotic potential exceeds the intracellular osmotic potential (Fig. 4B). Plotting the percentage of collapsed appressoria against the osmotic pressure of the PEG solution, a steep ascent of the graphs representing WT and Cgpks 1 strains can be observed between 2.0 and 3.5 MPa. Estimated $50 \%$ effective dose values deduced from the curve line fitted graphs were $2.4 \mathrm{MPa}$ for the WT and $2.9 \mathrm{MPa}$ for Cgpks 1 strains 2 and 16. Approximately 95\% of the appressoria of all strains had collapsed at 4.0 MPa (Fig. 4B).
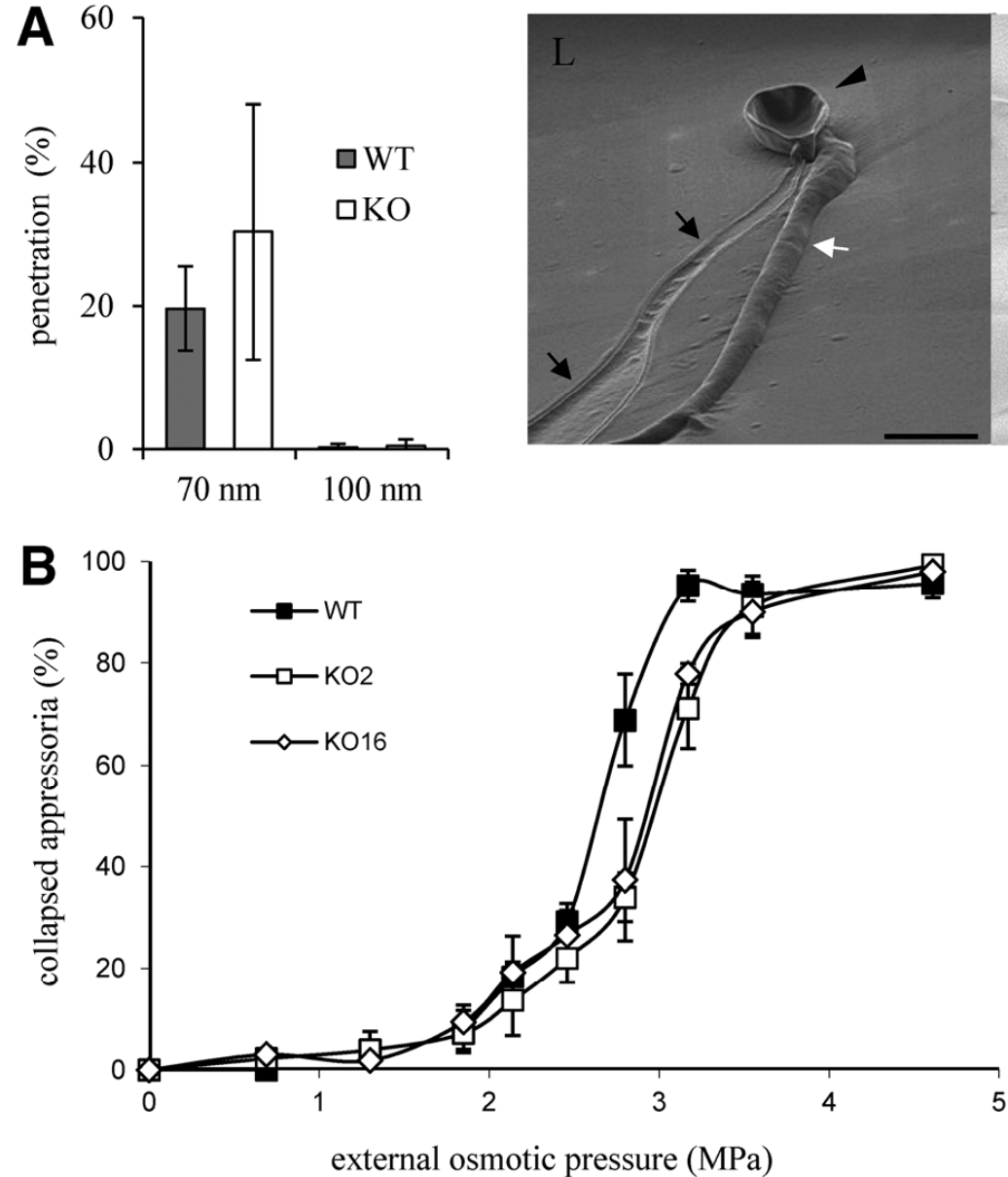
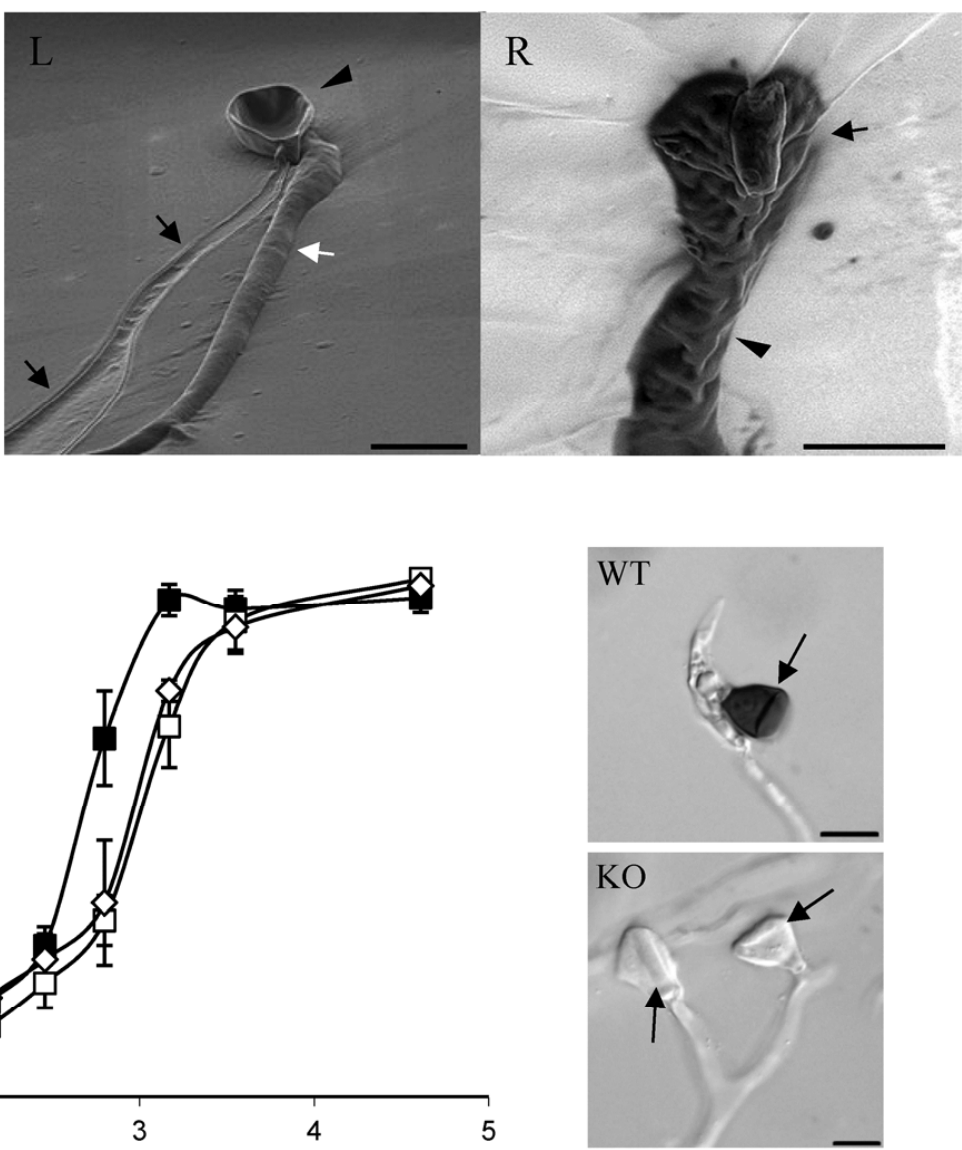

Fig. 4. Penetration competence and turgor generation. A, Penetration of ultrathin polytetrafluoroethylene (PTFE) membranes. Penetration efficiencies of Colletotrichum graminicola appressoria differentiated by the wild-type CgM2 (WT) and a melanin-deficient Cgpks 1 strain (knock-out [KO]) on nonbiodegradable PTFE membranes of highly defined thickness. PTFE layers of $70 \mathrm{~nm}$ were penetrated by the WT and the KO strain at rates that did not differ significantly. Rare penetrations events of appressoria formed by the WT and the KO strain occurred on PTFE layers of $100 \mathrm{~nm}$ but not 130 nm thickness ( $n=$ 450). Electron microscopy pictures were taken to illustrate the successful penetration of PTFE membranes by the KO strain. Left: view from above. Imprint of conidium and germ tube, which had been removed during sample preparation (black arrows). A black arrowhead indicates a collapsed appressorium after penetration of the PTFE layer. The underlying biotrophic hypha shapes the membrane (white arrow). Right: view from below. Fungal infection structures at the lower site of the PTFE membrane after successful penetration. A biotrophic hypha (arrowhead) emerges from the penetration site underneath the appressorium (arrow). Size bar $=10 \mu \mathrm{m}$. B, Determination of the appressorial turgor pressure by incipient-cytorrhysis. Increasing concentrations of polyethylene glycol-6000 induced cell collapse in appressoria of the WT and two individual Cgpks1 strains (KO2 and KO16). Compared with the WT, appressoria of the $\mathrm{KO}$ strains showed cytorrhysis at a slightly higher external osmotic potential, indicating similar osmolyte concentrations in appressoria of WT and KO strains. Micrographs were taken to visualize collapsed appressoria of the WT strain and a KO strain. Arrows point at the longitudinal cavity, a characteristic feature of collapsed appressoria $(n=300$, size bars $=10 \mu \mathrm{m})$. 
We used transmitted-light double-beam interference MachZehnder Microscopy (MZM) as a novel method to compare osmolyte concentrations in appressoria of Cgpks 1 mutants and the WT strain. Whereas incipient-cytorrhysis experiments require the incubation of cells in hyper-osmotic solutions, MZM allows observation and measurement of phase-shift phenomena in unstressed cells. Basically, phase shifts are indicative of changes in the concentration or nature of substances and, therefore, can be used to calculate the concentration of the dry mass to assess the osmotic potential of cells. Although the exact composition of appressorial solutes are not known for $C$. graminicola, WT and mutant appressoria can only be compared by MZM on the basis of the specific mean optical path differences (OPDs) (Loehrer et al. in press). Surprisingly, no significant differences in the mean specific OPD between WT and mutant appressoria were detected (Fig. 5), indicating that osmolytes are completely retained in the mutant appressoria, despite the lack of melanin. Because the precise composition of compounds in $C$. graminicola appressoria is as yet not known, we estimated the dry weight content of the WT and two mutant strains based on the refractive index increment value $(\mathrm{dn} / \mathrm{dc})$ of $0.151 \mathrm{ml} / \mathrm{g}$, which is specific for C6-sugar alcohols (Weast 1976). The dry mass content of WT appressoria was $436 \pm 40 \mathrm{~g} /$ liter and did not differ significantly from that of the Cgpksl strains.

In order to estimate the osmotic potential in appressoria, the dry weight content of the cytosol had to be calculated. This was achieved by subtracting the cell-wall fraction from the total fraction. It was shown in a previous study that the cell wall and plasma membrane fractions account for approximately $30 \%$ of the total dry weight content of M. oryzae and Phakopsora pachyrhizi (Loehrer et al. in press). Assuming a similar value for $C$. graminicola, an osmotic potential of approximately 3.9 MPa can be expected, based on the hypothesis that C6-sugar alcohols such as mannitol play a major role in appressoria of $C$. graminicola, as shown for appressoria of $M$. oryzae and $P$. pachyrhizi. The calculated osmotic potential is in agreement with the values obtained by incipient-cytorrhysis experiments. Taken together, these data strongly suggest that accumulation of osmolytes and generation of turgor in appressoria of $C$. graminicola is independent of melanin.

\section{Atomic force microscopy.}

Structural alterations in nonmelanized cell walls may lead to changes in mechanical properties. As a consequence, the application of techniques that depend on visible cell collapse (e.g., incipient-cytorrhysis assay) could be misleading. In order to visualize the morphology of the cell wall of Cgpksl and WT appressoria, atomic force microscopy (AFM) surface scans were performed at $10-\mathrm{nm}$ resolution. Unexpectedly, we observed considerable differences between appressorial cell walls of Cgpks1 and WT strains. WT appressoria showed a deeply indented surface whereas Cgpks 1 appressoria had a more smooth appearance, with less pronounced polygonal structures (Fig. 6). Although melanin apposition occurs as a thin layer directly adjacent to the cell membrane, it still seems to affect surface regions of the appressorial cell wall.

\section{DISCUSSION}

Penetration of intact host cuticles and epidermal cell walls by fungal species is an indispensable prerequisite for gaining access to nutrients, intracellular parasitic growth, and, hence, propagation. In many fungi, successful penetration depends primarily on melanized appressoria that function in turgor generation, firm attachment to the host surface, localized secretion of CWDE, as well as differentiation of the penetration peg
(Deising et al. 2000; Mendgen et al. 1996). Following the proposed function of melanin in establishing and maintaining high appressorial turgor pressure required for penetrating plant barriers, essential physiological and structural prerequisites of appressorial components and plant cell walls can be postulated. First, the appressorial plasma membrane does not constitute an effective barrier for small molecule osmolytes such as glycerol. Second, the melanin layer forms a meshwork which is tight enough to prevent solutes from passing through but wide enough to enable the influx of water molecules. Third, plant surfaces must have the same retention properties as the appressorial cell wall because appressorial pore and penetration peg are not melanized.

The hypothesis of melanin forming a tight meshwork in close vicinity of the cell membrane to prevent solutes from leaking into the apoplast, which would make high demands on consistent cross-linking of melanin precursors, is mainly based on indirect methods employed to determine pore size diameters and the osmotic potential in appressoria of M. oryzae. Therefore, we reexamined the ability of nonmelanized appressoria of a $C$. graminicola PKS1 disruption strain to generate intracellular turgor using standard methods and the previously described transmitted-light double-beam interference MZM.

It is obvious that constant loss of biomolecules which may serve as an energy source or precursors for polymers would be deleterious for living cells. Therefore, lipid bilayers constitute a barrier that largely blocks passive diffusion. Determination of diffusion coefficients of hydrated molecules such as urea and glycerol showed that only very small amounts of these solutes permeate through artificial phospholipid bilayers (Paula et al. 1996). Further experimental work supports the concept of highly regulated accumulation and release of glycerol by specific membrane transporters in the model organisms Schizosaccharomyces pombe and Saccharomyces cereviseae (Hohmann et al. 2007; Kayingo et al. 2004; Luyten et al. 1995).

Solute exclusion experiments by Howard and associates (1991) showed that concentrated solutions of PEGs of different molecular weights caused visible cell collapse but not plasmolysis. Because even PEG-200 caused cell collapse and, thus, failed to diffuse across the cell wall, the authors reasoned that the mature melanized appressorium wall of $M$. oryzae must have a pore size smaller than $1 \mathrm{~nm}$. Nonmelanized ap-

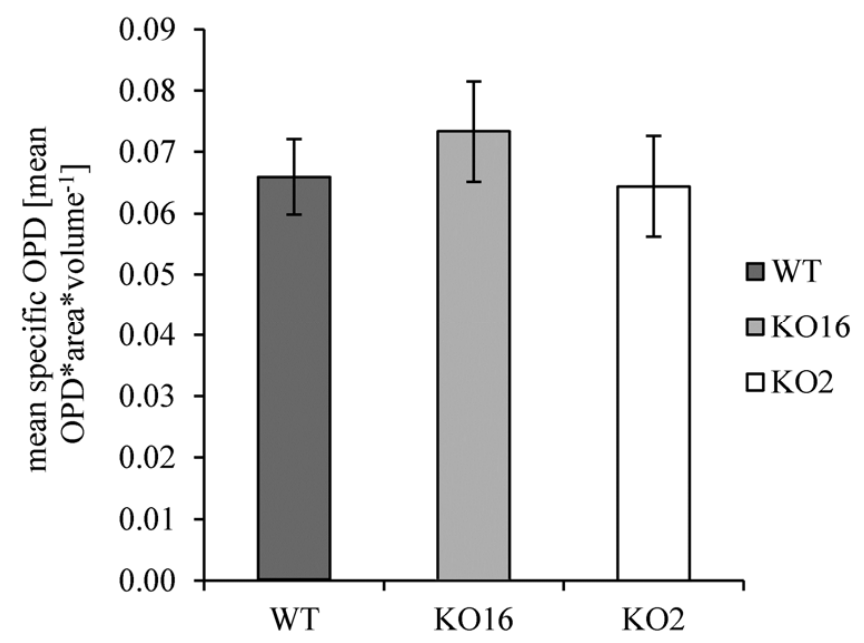

Fig. 5. In vivo measurement of the mean optical path density (OPD) in appressoria of Colletotrichum graminicola. The OPD in appressoria of the wild-type (WT) and two individual Cgpks1 strains (KO2 and KO16) was determined by Mach-Zehnder transmission-light interferometry using the phase shift algorithm. There was no significant difference between WT and $\mathrm{KO}$ appressoria indicating similar optical density and, thus, similar osmotic water potentials $(n=10)$. 
pressoria, in contrast, had an exclusion threshold between PEG-400 and PEG-600, corresponding to a pore diameter of 1 to $2 \mathrm{~nm}$. However, the effective hydrodynamic radii of glycerol, which has been described as the major osmolyte in appressoria of M. oryzae (De Jong et al. 1997), and PEG-200 are 0.31 and $0.43 \mathrm{~nm}$, respectively (Merzlyak et al. 1999).

In $C$. graminicola, we attempted to measure cell-wall pore sizes by incipient-cytorrhysis, applying increasing concentrations of PEG-200, -300, -400, and -600; glycerol; and $\mathrm{KCl}$. This approach, however, proved unsuccessful because plasmolysis could not be observed in either WT or mutant appressoria under all conditions tested.

As described for $M$. oryzae, nonmelanized appressoria of Cgpks 1 strains were unable to penetrate intact maize and onion epidermis cells but were not impaired in invasive growth and symptom development on wounded maize leaves (Fig. 2) (Rasmussen and Hanau 1989). In order to test whether Cgpks1 strains were generally defective in pressure-driven penetration, we performed penetration experiments on highly defined PTFE membranes. Unexpectedly, Cgpksl mutants of C. graminicola were able to penetrate nonbiodegradable PTFE membranes of 70 to $100 \mathrm{~nm}$ at rates comparable with those of the WT strain. These results clearly showed that Cgpksl strains are not generally impaired in pressure-driven penetration, and reveal differences in the penetration process of living cells and artificial membranes. Interestingly, albino strains of $C$. lagenarium complemented with the THN synthase gene PKS1 from Alternaria alternata were able to penetrate mylar membranes but not plants (Takano et al. 1997).

In order to test whether the generation of turgor pressure in Cgpks1 appressoria is compromised, analysis of incipient-cytorrhysis was performed. Supporting the data obtained with PTFE membranes, incipient-cytorrhysis did not provide any evidence for a reduced osmotic potential in nonmelanized appressoria of Cgpks1 mutant strains. These unexpected findings contrast the results of studies on melanin-deficient appressoria from other phytopathogenic fungi. Melanized appressoria of $M$. oryzae accumulate solutes with an osmotic potential of up to $8.0 \mathrm{MPa}$, which is 30 to $70 \%$ higher than in appressoria of melanin-deficient mutants of this fungus (Dean 1997). Similarly, nonmelanized appressoria of $C$. lindemuthianum showed cell collapse at much lower osmolyte concentrations than melanized appressoria (Veneault-Fourrey et al. 2005).

One could argue that melanin apposition may alter mechanical properties of the cell wall, thus biasing experimental results obtained by incipient-cytorrhysis assays. For example, appressoria of Phyllosticta ampelicida were highly resistant to collapse during periods of desiccation, probably due to a melanindependent increase in rigidity of the appressorium cell wall (Kuo and Hoch 1995). Hence, turgor estimates based on cytorrhysis may significantly overestimate appressorial turgor pressure (Howard and Valent 1996). In order to gain insight into the topography of appressorial cell walls of $C$. graminicola, we employed AFM at a $10-\mathrm{nm}$ resolution. One of the surprises of this study was that melanin apposition affects the surface structure of the appressorial cell wall, in spite of the fact that melanin is restricted to the inner appressorial cell wall. AFM surface scans showed significant structural differences between melanized and nonmelanized appressorial cell walls. A reason for this observation could be an altered diffusion of secreted fungal enzymes rather than a direct effect of melanin on the structure of the cell wall. Hence, enzymes involved in synthesis or modification of the appressorial cell wall could not diffuse across the melanin layer, which would support the concept of sequestration of CWDE activity by the melanin layer (Kubo and Furusawa 1986, 1991).

In order to circumvent methodical bias attributed to alterations in the mechanical properties of nonmelanized and melanized appressoria and to verify the results of the cell collapse assay, we applied the previously described transmittedlight double-beam interference MZM that allows noninvasive live-cell imaging of phase-shifting substances on a cellular

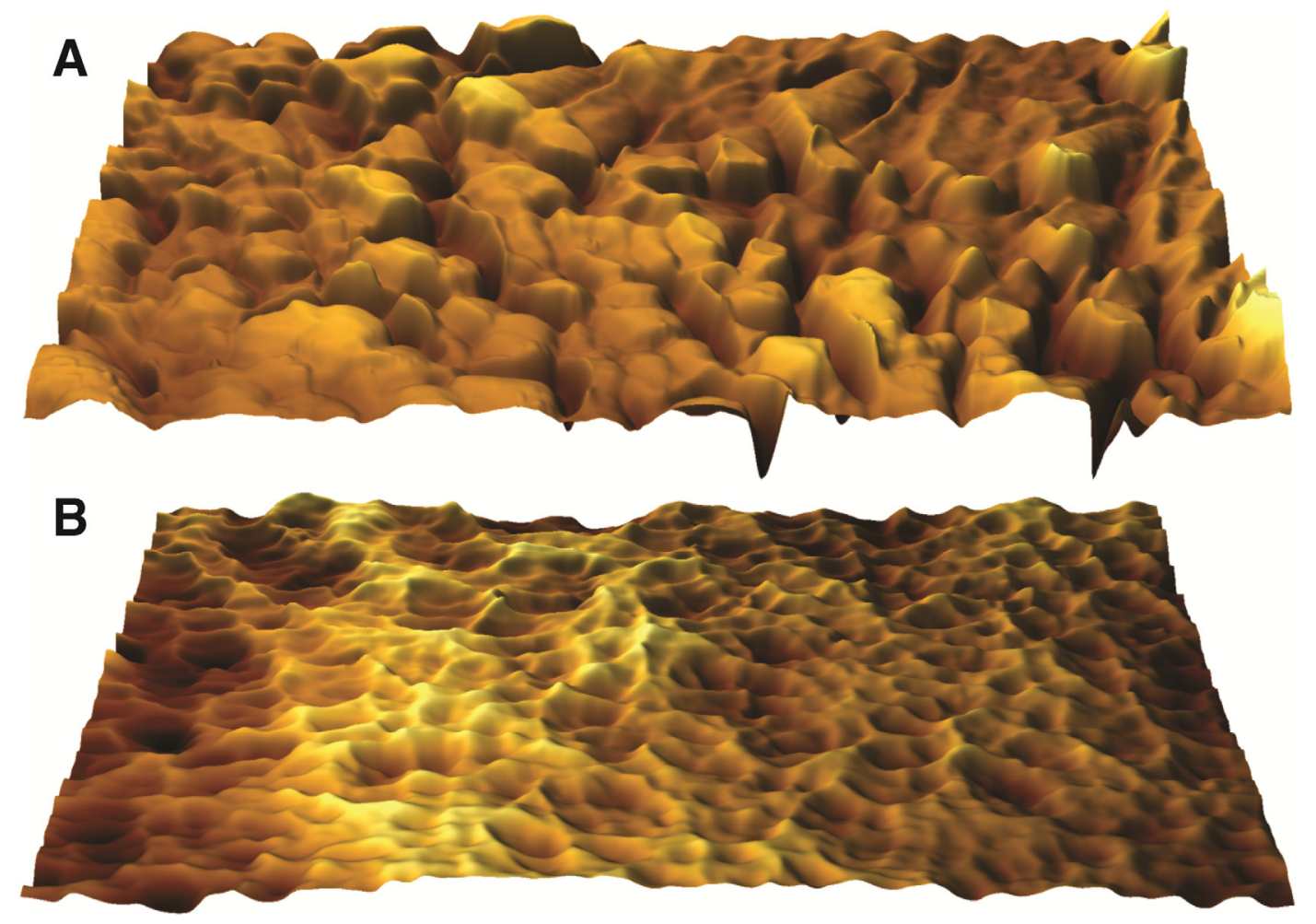

Fig. 6. Atomic force microscopy. Surface scans of A, melanized wild-type appressoria revealed a deeply indented profile, whereas B, nonmelanized Cgpks 1 appressoria displayed less pronounced elevations. X and Y dimensions are 2.0 by $1.27 \mu \mathrm{m} ; \mathrm{z}$ values for the WT and mutant are 162 and $100 \mathrm{~nm}$, respectively. 
level (Supplementary Fig. S6) (Loehrer et al. in press). MZM is a powerful technique that enables direct determination of osmolyte concentrations in living cells. Because the exact composition of substances in the appressorial cytosol of $C$. graminicola is not known, we used the mean specific OPD for comparison of WT and mutant appressoria. Melanized and nonmelanized appressoria did not show a significant difference in their OPD values. Moreover, the calculated osmotic potential of approximately $3.9 \mathrm{MPa}$ is in agreement with data obtained from incipient-cytorrhysis experiments. This result provides further evidence for generation of the full osmotic potential in Cgpksl appressoria and demonstrates the applicability of incipient- cytorrhysis for determination of turgor pressures in appressoria of $C$. graminicola. Taken together, the results obtained from PTFE membrane penetration, incipient-cytorrhysis assays, and MZM analyses did not reveal significant differences in the osmotic potential in appressoria of WT and Cgpks 1 mutant strains. Therefore, we conclude that melanization is not a requirement for osmolyte accumulation and turgor generation in appressoria of $C$. graminicola.

In search for alternative melanin functions, observation of lateral germination in melanin-deficient appressoria of $C$. lagenarium by Kubo and Furusawa (1986, 1991) suggest that melanin may be responsible for appressorial cell-wall rigidity, thereby focusing the turgor forces for vertical penetration or the secretion of fungal enzymes which may support penetration of host surfaces (Howard and Valent 1996; Howard et al. 1991). In addition, melanized cells have shown to be more resistant to enzymatic lysis (Howard and Ferrari 1989; Jacobson 2000). probably due to increased cross-linking. Indeed, Zhong and coworkers (2008) found labeled L-DOPA precursors to be linked to polysaccharides in cell walls of $C$. neoformans. Radical polymerization of melanin precursor molecules seems to include cell-wall components in a random process which results in a multitude of covalent bonds that are inaccessible to CWDE. Therefore, melanization may significantly contribute to cell-wall rigidity and, thus, resistance against enzymatic degradation.

To test this assumption, appressoria of WT and Cgpks 1 strains were subjected to hydrolytic degradation using lysing enzymes of $T$. harzianum. Approximately one-third of the in vitro differentiated mutant appressoria disintegrated whereas less than $3 \%$ of the WT appressoria were affected. Similar findings have been made in vivo on intact maize leaves, although effects were not as drastic as in the in vitro experiments. These results strongly suggest that melanization protects appressorial cell walls of $C$. graminicola against degradation by exogenous CWDE.

Increased rigidity may also be beneficial to protect appressorial cell walls against autolytic CWDE. Sublateral branching of fungal hyphae, for example, depends on the activity of secreted fungal CWDE which modify the plasticity of the cell wall, thus enabling differentiation of a new apex, a process that appears to be similar to the formation of the protruding penetration peg at the appressorial base. However, activity of autolytic CWDE may be deleterious for appressoria generating exceptional hydrostatic pressure. Therefore, melanization might be required for site-directed formation of the penetration peg at the nonmelanized penetration pore and confers increased resistivity to the melanized appressorial cell wall. In line with this, chemical complementation of Cgpks1 strains by scytalone restored pigmentation of appressoria; however, appressoria of the complemented isolates displayed lateral germination, were irregularly shaped, and ruptured frequently. Thus, precise regulation of synthesis and integration of melanin seems to be a prerequisite for rigidity of the cell wall whereas failures in this process lead to strongly reduced cell-wall stability and, hence, nonpathogenicity.
Our observations of lysed appressoria on intact maize leaves or onion epidermis cells may be equivalent to appressorium lysis of $M$. oryzae mutants lacking $\alpha-1,3$-glucan synthase (Fujikawa et al. 2012). The authors hypothesize that $\alpha-1,3-$ glucan is involved in the protection of the fungal cell wall against digestive enzymes secreted by plants during infection. Because epidermal cutin layers are supposed to prevent larger molecules from escaping the plant apoplast, this phenotype might be explained more likely by the activity of secreted fungal glucanases and chitinases. Secretion of fungal $\beta$-glucanases, for example, could be an instrument to degrade papillae which are often formed as a defense response. This enzymatic activity, however, would be deleterious to the rigidity of the fungal cell wall whose structure and resistivity depends largely on both $\beta$-glucans and chitin.

Taken together, apposition of melanin in the appressorial cell wall results in a melanin layer which provides protection against CWDE and helps prevent access of cell-wall-modifying enzymes to structurally relevant cell-wall polymers. Because this layer is localized directly adjacent to the cell membrane, one would expect a primary role as a barrier for fungusderived CWDE. However, exogenous application of CWDE caused lysis of nonmelanized appressoria whereas melanized appressoria were not affected, indicating a putative role also in protection against plant-derived CWDE which are part of the plant frontline defense. Site-specific differentiation of the penetration peg requires secretion of fungal enzymes which modify the plasticity of the appressorium cell wall, thus enabling differentiation of a new apex. Secretion of fungal CWDE at the site of attempted penetration may partially degrade the plant cell wall in order to support the pressure-driven penetration process.

Based on our experimental results, we propose a novel role for melanin in appressoria of $C$. graminicola. Localized melanization of the appressorium acts as an instrument to focus enzyme secretion, thereby determining the direction of penetration as shown by lateral germination of Cgpks1 appressoria. At the same time, melanin confers protection against fungal CWDE by preventing diffusion of these enzymes into the cellwall space and improves rigidity of the appressorium by crosslinking cell-wall components in the critical penetration phase, when high internal pressure coincides with the secretion of fungal CWDE and host defense mechanisms.

\section{MATERIALS AND METHODS}

\section{Fungal isolates, culture conditions, and maize infection assays.}

The WT strain CgM2 of $C$. graminicola was provided by R. L. Nicholson, Purdue University, West Lafayette, IN, U.S.A. Fourteen-day-old maize (Zea maize 'Golden Jubilee') plants, leaf segments (third leaf), or epidermal cell layers from onion bulbs (Allium cepa 'Grano') were used to assess virulence of $\mathrm{CgM} 2$ and transformants. Inoculation was performed with 10$\mu \mathrm{l}$ droplets containing $10^{3}$ conidia in $0.01 \%$ (vol/vol) Tween 20. Leaf segments $(8 \mathrm{~cm})$ were incubated in sealed petri dishes in darkness at $23^{\circ} \mathrm{C}$. Mock inoculation was done with sterile $0.01 \%$ (vol/vol) Tween 20. Plants were incubated in a Percival AR-75L growth chamber (Percival, Perry, IA, U.S.A.) (settings: $12 \mathrm{~h}$ of light, $200 \mu \mathrm{E}, 70 \%$ relative humidity, $25^{\circ} \mathrm{C}$ ).

\section{CWDE assay.}

To analyze the effect of CWDE activity on the integrity of appressorial cell walls, $10-\mu \mathrm{l}$ droplets containing $0.35 \mathrm{M} \mathrm{NaCl}$ and $5 \times 10^{4}$ conidia of either WT or Cgpks 1 mutant strains were pipetted onto hydrophobic polyester transparencies (Corporate Express, Stuttgart, Germany) and incubated in sealed 
petri dishes at $23^{\circ} \mathrm{C}$ for $24 \mathrm{~h}$. After formation of mature appressoria, T. harzianum Lysing Enzymes (Sigma-Aldrich, Munich, Germany) were added in a final concentration of $5 \mathrm{mg} / \mathrm{ml}$ and samples were incubated at $23^{\circ} \mathrm{C}$ for 12 to $24 \mathrm{~h}$ prior to microscopic evaluation.

\section{Extraction of RNA, qRT-PCR, and whole-genome transcriptome profiling (RNA-seq).}

To perform qRT-PCR, total RNA was extracted from 14-dayold maize plants (third leaf) sprayed with $10^{6}$ conidia $/ \mathrm{ml}$ using the Peq Gold RNA extraction kit (Peqlab, Erlangen, Germany) with lysis buffer QC (Qiagen, Hilden, Germany). Samples were collected at $0,12,24,36,48,72,96$, and 120 hpi. Genespecific primers employed to analyze transcript abundance of the $C g P K S 1$ gene were GLRG_04203 for (TCAATCTGAGCC CTGGACAC) and GLRG_04203 rev (GTCCTGAGATCGCC AAGCTG). Transcript levels of the $C$. graminicola Histone $\mathrm{H} 3$ gene were used for normalization (primers GGAGGTCGGAC TTGAAGTCCT and CGAGATCCGTCGCTACCAGA). qPCR was performed essentially as described in Horbach and associates (2009). Threshold cycle values of three biological replicates were used to calculate average values and standard deviations according to Pfaffl (2001). All values are standardized to the average threshold cycle value obtained with RNA extracted from maize leaves that were inoculated with $\mathrm{CgM} 2$ at time point 0 hpi.

To screen for the presence of $C g P K S 1$ transcripts in individual Cgpks 1 strains, primers CgPKS1fl (ATGGCCGATATGAT GTCTTATCTCC) and CgPKSlint1rev (GCTCCTTCTCGGT GCGGTCG) were used in RT-PCRs. Primers for the constitutively expressed chitin synthase $C g C H S I I$ gene were CHSIII and CHSII3 (Werner et al. 2007). Total RNA (50 ng) pretreated with RQ1 RNase-free DNase (Promega Corp., Madison, WI, U.S.A.) was used in 10- $\mu$ l RT-PCR reactions (One Step RT-PCR kit; Qiagen). Following synthesis of $\mathrm{cDNA}$ at $50^{\circ} \mathrm{C}$ for $30 \mathrm{~min}$, 25 cycles comprising initial denaturation $\left(95^{\circ} \mathrm{C}, 3 \mathrm{~min}\right)$, denaturation $\left(95^{\circ} \mathrm{C}, 30 \mathrm{~s}\right)$, annealing $\left(60^{\circ} \mathrm{C}, 20 \mathrm{~s}\right)$, and extension $\left(72^{\circ} \mathrm{C}\right.$, $50 \mathrm{~s})$ yielded the expected $C g P K S 1 / C g C H S I I$ products for the WT whereas the $C g P K S 1$ but not the $C g C H S I I$ product was absent in three independent Cgpks 1 strains.

For RNA-seq, maize plants were grown in individual pots for 14 days at $23^{\circ} \mathrm{C}$. The third leaf was carefully fixated in a horizontal position and inoculated with $10-\mu$ d droplets containing $10^{4}$ conidia in $0.01 \%$ ( $\mathrm{vol} / \mathrm{vol}$ ) Tween 20 . After $24 \mathrm{~h}$ in a moisture chamber at $25^{\circ} \mathrm{C}$, plants were transferred to an environmentally controlled greenhouse cabinet and incubated at $25^{\circ} \mathrm{C}$. Samples corresponding to three biological replicates were taken at $0,12,24,48,72$, and 120 hpi using a cork borer (diameter of $8 \mathrm{~mm}$ ).

RNA quality and quantity were examined using a NanoDrop 1000 photometer (Peqlab, Erlangen, Germany) and Bioanalyzer 2100 (Agilent, Böblingen, Germany) according to the manufacturer's instructions. RNA samples were further processed and sequenced at ServiceXS (Leiden, Netherlands).

Raw reads were mapped to the current genome annotation of $C$. graminicola using Tophat 2 from the Broad Institute website (Kim et al. 2011; Trapnell et al. 2012) with standard parameters except for minimum intron size, which was set to 30 . Mapped reads were counted using HTSeq. Differential gene expression was analyzed using the $\mathrm{R} /$ Bioconductor package DESeq (Anders and Huber 2010).

\section{DNA extraction and $C g P K S 1$ isolation.}

Genomic DNA was isolated from vegetative mycelia of $C$. graminicola following the method described by Döbbeling and associates (1997). Starting from a previously isolated PKS gene fragment with similarity to fungal PKS involved in pigment biosynthesis (Sugui and Deising 2002), the complete DNA sequence of $C g P K S 1$ plus approximately $5 \mathrm{~kb}$ of DNA sequence information upstream and downstream of the deduced ORF were obtained by genome walking according to Liu and Baird (2001)

Sequence analysis of PCR products derived from genomic DNA and cDNA revealed the intron/exon structure of $C g P K S 1$.

\section{Targeted inactivation of $\mathrm{CgPKS1}$} and complementation of the Cgpks1 strain.

A gene disruption construct was designed to abolish CgPKS1 expression by double-crossover integration of an $\mathrm{hph}$ resistance cassette into the ORF. First, an internal 1.7-kb fragment of $C g P K S 1$ was amplified using the primers CgPKS1KO1 (CAACGCTGCATACCTCCG) and CgPKS1KO2 (CGCGCAT CCTGCCGGGGAGAC) and cloned into pGEM-T Easy (Promega, Mannheim, Germany). The resulting plasmid pGEM CgPKS1KO was cleaved at the unique Bsp119I restriction site. A 3.7-kb DNA fragment containing the pAN7-1 (GenBank accession number Z32698) $h p h$ resistance cassette was excised from vector pGEM hph Bsp119I by Bsp119I digestion and cloned into pGEM CgPKS1KO. The disruption construct was amplified by PCR using primers CgPKS1KO1/2 and transferred into conidial protoplasts as described (Werner et al. 2007).

For genetic complementation, the $C g P K S 1$ genomic sequence, including promoter and terminator regions, was amplified by PCR and used for transformation of Cgpks 1 protoplasts. In addition, a second construct spanning the $C g P K S 1$ coding region and terminator was fused to the constitutive oliC promoter from Aspergillus nidulans (Ward and Turner 1986) and amplified by PCR. Both PCR products were co-transformed with the nourseothricine resistance cassette (primers noursf CCGCTCTAGAGCCGCATTC and noursr AAGCTTG ATATCTGTTAGTAATCATCATTAAG, amplified from vector pNR) (Malonek et al. 2004).

\section{Southern blot analysis.}

To perform genomic Southern blot analysis, $10 \mu \mathrm{g}$ of XhoIdigested DNA was separated on a $0.8 \%$ (wt/vol) agarose gel in Tris-acetate-EDTA buffer, depurinated, and blotted onto a positively charged nylon membrane (Hybond-N+; Amersham Pharmacia Biotech, Freiburg, Germany) by downward alkaline capillary transfer (Brown 1999). Specific primers CgPKS1 probe1 (CGCGTCAGCTACCTTATCAAG) CgPKS1 probe2 (GGTACCGTTGATGATGAAGCC) and plasmid pGEM CgPKS1KO as template DNA were used to generate a 284-bp alkali-labile digoxigenin-dUTP-labeled probe (Roche Diagnostics, Mannheim, Germany). Hybridization and probe detection followed the recommended protocol. The membrane was exposed to Hyperfilm ECL X-ray film (Amersham Pharmacia Biotech, Freiburg, Germany).

\section{Construction of the $C g P K S 1: G F P$ fusion construct.}

A PCR with primers PKSEGFP1 (ACCGGTACTCCCCGC GGATCGGTG; AgeI site underlined) and PKSEGFP2 (TTCG AAAACGTACTCGATGTAGTGAGC; Bsp119I site underlined) yielded a $1.3-\mathrm{kb}$ fragment containing $300 \mathrm{bp}$ of the $C g P K S 1$ ORF and $1 \mathrm{~kb}$ of the promoter region. Cloning in pGEM-T Easy (Promega) resulted in plasmid pPKS1egfp1. The unique Bsp119I site was then used to fuse a $1.5-\mathrm{kb}$ PCR fragment containing the GFP ORF and terminator TrpC (primers EGFP3 TTTTCGAAATGGTGAGCAAGGGCGAG, Bsp119I site underlined; and EGFP4 TTTTCGAACGCAGGACAATCGCTA CAG, Bsp119I site underlined) from vector pSH1.51EGFP in frame with $C g P K S 1$. To introduce a resistance marker, the 2.4-kb $h p h$ cassette of pCR2.1HPHAgeI ( $h p h$ cassette of plas- 
mid vector pAN7-1 flanked by AgeI sites in plasmid pCR2.1) was excised and cloned into the corresponding AgeI site of the GFP fusion vector. The complete fusion construct was excised from pGEM-T Easy by NotI digestion, purified by gel elution, and transferred into conidial protoplasts as described (Werner et al. 2007).

\section{Protein purification and \\ 4'-phosphopantetheinylation assay.}

Heterologous expression and purification of CgPPT1 and the ACP domain of CgPKS1was performed as described by Horbach and associates (2009). Protein 4'-phosphopantetheinylation reactions were performed with biotin-conjugated CoA (Covalys, Witterswil, Switzerland). Labeling reactions were analyzed by sodium dodecyl sulfate-polyacrylamide gel electrophoresis. Biotinylated (His) ${ }_{6}$-CgPKS $1 f$ was detected using hrp-conjugated streptavidin (Pierce Biotechnology, Rockford, IL, U.S.A.), essentially as described (Yin et al. 2005).

\section{Staining procedures and microscopy.}

Nile Red staining solution (Nile Red at $2 \mu \mathrm{g} / \mathrm{ml}$ in phosphate buffered saline $[\mathrm{PBS}]$ ) was prepared from an acetone stock solution containing Nile Red at $500 \mu \mathrm{g} / \mathrm{ml}$. Conidia or infection structures differentiated on polyester sheets or glass slides were incubated for $5 \mathrm{~min}$ in Nile Red staining solution and washed twice in PBS (3.2 mM Na $2 \mathrm{HPO}_{4}, 0.5 \mathrm{mM} \mathrm{KH_{2 }} \mathrm{PO}_{4}$, $1.3 \mathrm{mM} \mathrm{KCl}$, and $135 \mathrm{mM} \mathrm{NaCl}, \mathrm{pH}$ 7.4).

Calcofluor White stock solution was prepared as recommended by the manufacturer (Sigma-Aldrich, München, Germany). Fungal structures were stained for $15 \mathrm{~min}$ in PBS containing Calcofluor White at $35 \mu \mathrm{g} / \mathrm{ml}$ and subsequently washed twice in PBS prior to microscopy.

Aniline blue (Carl Roth, Karlsruhe, Germany) was dissolved in lactophenol $(40 \%$ [vol/vol] glycerol, $20 \%$ [vol/vol] phenol, $20 \%$ [vol/vol] lactic acid, and $0.0025 \%$ [wt/vol] aniline blue). Samples were incubated for $3 \mathrm{~h}$ at $80^{\circ} \mathrm{C}$ and destained in ethanol/chloroform (3:1) at room temperature.

Fluorescence microscopy was carried out as described in Horbach and associates (2009).

Scanning electron microscopy was used to visualize penetration of PTFE membranes essentially as described by Küster and associates (2008).

\section{PTFE penetration, cytorrhysis assays, and Mach-Zehnder interferometry.}

PTFE membranes of defined thickness were prepared as described (Küster et al. 2008). In order to separate films from glass slides, the potassium bromide layer was dissolved in distilled water, and PTFE layers floated as a membrane film carried by the surface tension of the water. Droplets of spore suspension containing $1 \times 10^{5} \mathrm{conidia} / \mathrm{ml}$ were carefully pipetted onto the membrane and incubated in sealed petri dishes at $23^{\circ} \mathrm{C}$ for $48 \mathrm{~h}$. The penetration frequency of appressoria formed on PTFE layers of 70 to $130 \mathrm{~nm}$ was determined microscopically in five biological replicates.

Appressorial turgor was measured by incipient cytorrhysis essentially as described by Horbach and associates (2009). Osmotic pressures of PEG solutions were calculated according to Money (1989). The numbers of collapsed and intact appressoria of five individual plastic sheets were counted for each concentration using light microscopy.

Conidia for Mach-Zehnder transmission-light interferometry (MZM) were suspended in sterile distilled water and transferred onto microscopy slides. The glass slides were incubated for $24 \mathrm{~h}$ in a dark moisture chamber at $22^{\circ} \mathrm{C}$. The cover slides were sealed with nail varnish prior to analysis to prevent evapo- ration. A reference slide without spores was prepared in parallel. MZM analysis and data analysis were conducted as described by Loehrer and associates (in press). OPDs of 10 individual appressoria were determined. Dry mass concentrations were calculated based on a dn/dc value of 0.151 , which is specific for mannitol. The osmotic pressure in appressoria was estimated by substituting the molar concentration (which was calculated on basis of dry mass concentration and the molar mass of Mannitol) into the Van't Hoff equation.

\section{AFM.}

AFM images were obtained by means of a JPK Nanowizard II (JPK-Instruments, Berlin) using standard silicon nitride cantilever (Bruker, Billerica, MA U.S.A.) with a nominal force constant $\mathrm{k}_{\mathrm{c}}$ of $0.1 \mathrm{~N} / \mathrm{m}$ and a tip radius of approximately $10 \mathrm{~nm}$. AFM scans were conducted using an intermittent contact mode and microscopy glass slides with adhered appressoria kept in a liquid enclosure (SmallCell; JPK-Instruments) filled with double-distilled water at $25^{\circ} \mathrm{C}$.

\section{Statistics and calculations.}

Calculations ( $t$ test, $F$ test, analysis of variance, and Tukey's test) were done with the software XLSTAT (version 2013.5.02; STATCON, Witzenhausen, Germany).The Phylogenetic tree was constructed using ClustalW2.

\section{ACKNOWLEDGMENTS}

This work was supported by a research grant of the Deutsche Forschungsgemeinschaft (DFG) to R. Horbach and H. B. Deising (grant 585699) and by the Interdisciplinary Center for Crop Plant Research (IZN) of the University of Halle-Wittenberg. M. Löhrer was supported by an RWTH University grant in the frame of "Förderung des wissenschaftlichen Nachwuchses". We thank E. Vollmer and A. Beutel for excellent technical assistance; F. Weihmann and J. Teutschbein for providing RNA; A. Cismak for kind support in performing electron microscopy; and Y. Kubo, Graduate School of Life and Environmental Sciences, Kyoto for kindly providing scytalone.

\section{LITERATURE CITED}

Anders, S., and Huber, W. 2010. Differential expression analysis for sequence count data. Genome Biol. 11:R106.

Bastmeyer, M., Deising, H. B., and Bechinger, C. 2002. Force exertion in fungal infection. Annu. Rev. Biophysiol. 31:321-341.

Bechinger, C., Giebel, K.-F., Schnell, M., Leiderer, P., Deising, H. B., and Bastmeyer, M. 1999. Optical measurements of invasive forces exerted by appressoria of a plant pathogenic fungus. Science 285:1896-1899.

Bell, A. A., and Wheeler, M. H. 1986. Biosynthesis and functions of fungal melanins. Annu. Rev. Phytopathol.24:411-451.

Bergstrom, G. C., and Nicholson, R. L. 1999. The biology of corn anthracnose. Plant Dis. 83:596-608.

Brown, T. 1999. Southern blotting. Pages 2.9.1-2.9.15 in: Current Protocols in Molecular Biology. F. M. Ausubel, R. Brent, R. E. Kingston, D. D. Moore, J. G. Seidman, J. A. Smith, and K. Struhl, eds. John Wiley \& Sons, New York.

Butler, M. J., and Day, A. W. 1999. Fungal melanins: A review. Can. J. Microbiol. 44:1115-1136.

Chai, L. Y. A., Netea, M. G., Sugui, J., Vonk, A. G., van de Sande, W. W. J., Warris, A., Kwon-Chung, K. J., and Kullberg, B., J. 2010. Aspergillus fumigatus conidial melanin modulates host cytokine response. Immunobiology 215:915-920.

Dean, R. A. 1997. Signal pathways and appressorium morphogenesis. Annu. Rev. Phytopathol. 35:211-234.

Deising, H. B., Werner, S., and Wernitz, M. 2000. The role of fungal appressoria in plant infection. Microbes Infect. 2:1631-1641.

De Jong, J. C., McCormack, B. J., Smirnoff, and Talbot, N. J. 1997. Glycerol generates turgor in rice blast. Nature 389:244-245.

Doebbeling, U., Böni, R., Häffner, A., Dummer, R., and Burg, G. 1997. Method for simultaneous RNA and DNA isolation from biopsy material, culture cells, plants and bacteria. Biotechniques 22:88-90.

Elovson, J., and Vagelos, P. R. 1968. Acyl carrier protein. J. Biol. Chem. 243:3603-3611.

Freeman, J, and Ward, E. 2004. Gaeumannomyces graminis, the take-all 
fungus and its relatives. Mol. Plant Pathol. 5:235-252.

Fujikawa, T., Sakaguchi, A., Nishizawa, Y., Kouzai, Y., Minami, E., Yano, S., Koga, H., Meshi, T., and Nishimura, M. 2012. Surface $\alpha$-1,3-glucan facilitates fungal stealth infection by interfering with innate immunity in plants. PLoS Pathog. 8:e1002882.

Gachomo, E. W., Seufferheld, M. J., and Kotchoni, S. O. 2010. Melanization of appressoria is critical for the pathogenicity of Diplocarpon rosae. Mol. Biol. Rep. 37:3583-3591.

Henson, J. M., Butler, M. J., and Day, A. W. 1999. The dark side of the mycelium: Melanins of phytopathogenic fungi. Annu. Rev. Phytopathol. 37:447-471.

Hignett, R. C., Roberts, A. L., and Carder, J. H. 1978. The properties and extracellular enzymes of Venturia inequalis and their association with loss of virulence of the fungus in culture. J. Gen. Microbiol. 110:67-75.

Hohmann, S., Krantz, M., and Norlander, B. 2007. Yeast osmoregulation. Methods Enzymol. 428:29-45.

Horbach, R., Graf, A., Weihmann, F., Antelo, L., Mathea, S., Liermann, J. C., Opatz, T., Thines, E., Aguirre, J., and Deising, H. B. 2009. Sfp-type $4^{\prime}$-phosphopantetheinyl transferase is indispensable for fungal pathogenicity. Plant Cell 21:3379-3396.

Horbach, R., Navarro-Quesadac, A. R., Knogge, W., and Deising, H. B. 2011. When and how to kill a plant cell: Infection strategies of plant pathogenic fungi J. Plant Physiol. 168:51-62.

Howard, R. J., and Ferrari, M. A. 1989. Role of melanin in appressorium function, Exp. Mycol. 13:403-418.

Howard, R. J., and Valent, B. 1996. Breaking and entering: Host penetration by the fungal rice pathogen Magnaporthe grisea. Annu. Rev. Microbiol. 50:491-512.

Howard, R. J., Ferrari, M. A., Roach, D. H., and Money, N. P. 1991. Penetration of hard substrates by a fungus employing enormous turgor pressures. Proc. Natl. Acad. Sci. U.S.A. 88:11281-11284.

Idnurm, A., and Howlett, B. J. 2001. Pathogenicity genes of phytopathogenic fungi. Mol. Plant Pathol. 2:241-255.

Jacobson, E. S. 2000. Pathogenic roles for fungal melanins. Clin. Microbiol. Rev. 13:708-717.

Kayingo, G., Sirotkin, V., Hohmann, S., and Prior, B. 2004. Accumulation and release of the osmolyte glycerol is independent of the putative MIP channel Spac977.17p in Schizosaccharomyces pombe. Antonie Leeuwenhoek 85:85-92.

Kelly, C., Osbourn, A. E., and Caten, C. E. 1997. The genetics of Gaeumannomyces graminis with particular reference to pigment production and pathogenicity. Fungal Genet. Newsl. 44A:108.

Kim, D., Pertea, G., Trapnell, C., Pimentel, H., Kelley, R., and Salzberg, S. L. 2013. TopHat2: Accurate alignment of transcriptomes in the presence of insertions, deletions and gene fusions. Genome Biol. 14:R36.

Kubo Y., and Furusawa I. 1986. Localization of melanin in appressoria of Colletotrichum lagenarium. Can. J. Microbiol. 32:280-282.

Kubo Y., and Furusawa I. 1991. Melanin biosynthesis: Prerequisite for successful invasion of the plant host by appressoria of Colletotrichum and Pyricularia. Pages 205-218 in: The Fungal Spore and Disease Initiation in Plants and Animals. G. T. Cole, and H. C. Hoch, eds. Plenum Publishing, New York.

Kuo, K. C., and Hoch, H. C. 1995. Visualization of the extracellular matrix surrounding pycnidiospores, germlings, and appressoria of Phyllosticta ampelicida. Mycologia 87:759-771.

Küster, S., Ludwig, N., Willers, G., Hoffmann, .J, Deising, H. B, and Kiesow, A. 2008 Thin PTFE-like membranes allow characterizing germination and mechanical penetration competence of pathogenic fungi. Acta Biomater. 4:1809-1818.

Liu, G. Y., and Nizet, V. 2009. Color me bad: Microbial pigments as virulence factors. Trends Microbiol. 17:406-413.

Liu, O. W., Chun, C. D., Chow, E. D., Chen, C., Madhani, H. D., and Noble, S. M. 2008. Systematic genetic analysis of virulence in the human fungal pathogen Cryptococcus neoformans. Cell 135:174-188.

Liu, X., and Baird, W. V. 2001. Rapid amplification of genomic DNA ends by NlaIII partial digestion and polynucleotide tailing. Plant Mol. Biol. Rep. 19:261-267.

Loehrer, M., Botterweck, J., Jahnke, J., Mahlmann, D. M., Gaetgens, J., Oldiges, M., Horbach, R., Deising, H. B., and Schaffrath, U. In vivo assessment of the invasive force exerted by the Asian soybean rust fungus by resurrection of double-beam interferometry. New Phytol. In press.

Luyten, K., Albertyn, J., Skibbe, W., Prior, B. A., Ramos, J., Thevelein, J. M., and Hohmann, S. 1995. Fps1, a yeast member of the MIP family of channel proteins, is a facilitator for glycerol uptake and efflux and it is inactive under osmotic stress. EMBO (Eur. Mol. Biol. Organ.) J. 14:13601371.

Malonek, S., Rojas, M. C., Hedden P., Gaskin, P., Hopkins, P., and Tudzynski, B. 2004. The NADPH-cytochrome P450 reductase gene from Gibberella fujikuroi is essential for gibberellin biosynthesis. J. Biol.
Chem. 279:25075-25084.

Mednick, A. J., Nosanchuk, J. D., and Casadevall, A. 2005. Melanization of Cryptococcus neoformans affects lung inflammatory responses during cryptococcal infection. Infect. Immun. 73:2012-2019.

Mendgen, K., Hahn, M., and Deising, H. B. 1996. Mechanisms and morphogenesis of penetration by plant pathogenic fungi. Annu. Rev. Phytopathol. 34:367-386

Merzlyak, P. G., Yuldasheva, L. N., Rodrigues, C. G., Carneiro, C. M., Krasilnikov, O. V., and Bezrukov, S. M. 1999. Polymeric nonelectrolytes to probe pore geometry: Application to the alpha-toxin transmembrane channel. Biophys. J. 77:3023-3033.

Miner, C. S., Dalton, N. N. 1953. Glycerol. Page 279 in: American Chemical Society Monogr. No. 117, Reinhold Publishing Corp., New York.

Money, N. P. 1989. Osmotic pressure of aqueous polyethylene glycols. Relationship between molecular weight and vapor pressure deficit. Plant Physiol. 91:766-769.

Money, N. P. 1995. Turgor pressure and the mechanics of fungal penetration. Can. J. Bot. 73:96-102.

Money, N. P., Caesar-Ton That, T. C., Frederick, B., and Henson, J. M. 1998. Melanin synthesis is associated with changes in hyphopodialturgor, permeability, and wall rigidity in Gaeumannomyces graminis var. graminis. Fungal Genet. Biol. 24:240-251.

Nosanchuk, J. D., and Casadevall, A. 2006. Impact of melanin on microbial virulence and clinical resistance to antimicrobial compounds. Antimicrob. Agents Chemother. 50:3519-3528.

Paula, S., Volkov, A. G., Van Hoek, A. N., Haines, T. H., and Deamer, D. W. 1996. Permeation of protons, potassium ions, and small polar molecules through phospholipid bilayers as a function of membrane thickness. Biophys. J. 70:339-348.

Pfaffl, M. W. 2001. A new mathematical model for relative quantification in real-time RT-PCR. Nucleic Acids Res. 29:2002-2007.

Rasmussen, J. B., and Hanau, R. M. 1989. Exogenous scytalone restores appressorial melanization and pathogenicity in albino mutants of Colletotrichum graminicola. Can. J. Plant Pathol. 11:349-352.

Revankar, S. G., and Sutton, D. A. 2010. Melanized fungi in human disease. Clin. Microbiol. Rev. 23:884-928.

Steiner, U., and Oerke, E. C. 2007. Localized melanization of appressoria is required for pathogenicity of Venturia inaequalis. Phytopathology 97:1222-1230.

Sugui, J. A., and Deising, H. B. 2002. Isolation of infection-specific sequence tags expressed during early stages of maize anthracnose disease development. Mol. Plant Pathol. 3:19-203.

Takano, Y., Kubo, Y., Kawamura, C., Tsuge, T., and Furusawa, I. 1997. The Alternaria alternata melanin biosynthesis gene restores appressorial melanization and penetration of cellulose membranes in the melanindeficient albino mutant of Colletotrichum lagenarium. Fungal Genet. Biol. 21:131-140.

Thywißen, A., Heinekamp, T., Dahse, H.-M., Schmaler-Ripcke, J., Nietzsche, S., Zipfel, P. F., and Brakhage, A. A. 2011. Conidial dihydroxynaphthalene melanin of the human pathogenic fungus Aspergillus fumigatus interferes with the host endocytosis pathway. Front. Microbiol. 2:96.

Tian, S., Garcia-Rivera, J., Yan, B., Casadevall, A., and Stark, R. E. 2003. Unlocking the molecular structure of fungal melanin using ${ }^{13} \mathrm{C}$ biosynthetic labeling and solid-state NMR. Biochemistry 42:81058109

Trapnell, C., Roberts, A., Goff, L., Pertea, G., Kim, D., Kelley, D. R., Pimentel, H., Salzberg, S. L., Rinn, J. L., and Pachter, L. 2012. Differential gene and transcript expression analysis of RNA-seq experiments with TopHat and Cufflinks. Nat. Protocols 7:562-578.

Veneault-Fourrey, C., Lange, R., Lauge, T., and Langin, T. 2005. Nonpathogenic strains of Colletotrichum lindemuthianum trigger progressive bean defense responses during appressorium-mediated penetration. Appl. Environ. Microbiol. 71:4761-4770.

Volling, K., Thywissen,, A., Brakhage, A. A., and Saluz, H. P. 2011. Phagocytosis of melanized Aspergillus conidia by macrophages exerts cytoprotective effects by sustained PI3K/Akt signalling. Cell Microbiol. 13:1130-1148.

Walsh, C. T., Gehring, A. M., Weinreb, P. H., Quadri, L. E., and Flugel, R. S. 1997. Posttranslational modification of peptide synthases polyketide and nonribosomal peptide synthases. Curr. Opin. Chem. Biol. 1:309315.

Wang, Y., Aisen, P., and Casadevall, A. 1995. Cryptococcus neoformans melanin and virulence: Mechanism of action. Infect. Immun. 63:31313136 .

Ward, M., and Turner, G. 1986. The ATP synthase subunit 9 gene of Aspergillus nidulans: Sequence and transcription. Mol. Gen. Genet. 205:331-338

Weast, R. C. 1976. Handbook of Chemistry and Physics, 57th ed. R. C. Weast, ed. CRC Press, Cleveland. 
Werner, S., Sugui, J. A., Steinberg, G., and Deising, H. B. 2007. Achitin synthase with a myosin-like motor domain is essential for hypha growth, appressorium differentiation and pathogenicity of the maize anthracnose fungus Colletotrichum graminicola. Mol. Plant-Microbe Interact. 20:1555-1567.

Yin, J., Straight, P. D., McLoughlin, S. M., Zhou, Z., Lin, A. J., Golan, D. E. Kelleher, N. L., Kolter, R., and Walsh, C. T. 2005. Genetically encoded short peptide tag for versatile protein labeling by Sfp phosphopantetheinyl transferase. Proc. Natl. Acad. Sci. U.S.A. 102:15815-15820.

Youngchim, S., Pornsuwan, S., Nosanchuk, J. D., Dankai, W., and Vanittanakom, N. 2011. Melanogenesis in dermatophyte species in vitro and during infection. Microbiology 157:2348-2356.
Zhong, J., Frases, S., Wang, H., Casadevall, A., and Stark, R. E. 2008. Following fungal melanin biosynthesis with solid-state NMR: Biopolymer molecular structures and possible connections to cell-wall polysaccharides. Biochemistry 47:4701-4710.

\section{AUTHOR-RECOMMENDED INTERNET RESOURCES}

ClustalW2 database:

www.ebi.ac.uk/Tools/phylogeny/clustalw2_phylogeny

Broad Institute website: www.broadinstitute.org

HTSeq database: www-huber.embl.de/users/anders/HTSeq 